\title{
МАТЕМАТИЧЕСКАЯ МОДЕЛЬ АВТОКОЛЕБАНИЙ В СИСТЕМЕ ЦИСТЕИН - ОКСИГЕНИРОВАННЫЕ КОМПЛЕКСЫ КОБАЛЬТА (II) С о-ДИСАЛИЦИЛИДЕНФЕНИЛЕНДИАМИНОМ И ЦИТОЗИНОМ
}

\begin{abstract}
С.С. Етмишева, У.Г. Магомедбеков, У.Г. Гасангаджиева, Х.М. Гасанова
СОФИЯ СЕЙПУЛЛАЕВНА ЕТМИШЕВА - ст. преподаватель кафедры неорганической химии Дагестанского государственного университета. E-mail: sophi05@yandex.ru.

УХУМААЛИ ГАДЖИЕВИЧ МАГОМЕДБЕКОВ - д.х.н., профессор, заведуюший кафедрой неорганической химии Дагестанского государственного университета. E-mail: ukhgmag@таil.ru.

УМУКУСУМ ГУСЕЙНОВНА ГАСАНГАДЖИЕВА - к.Х.н.,, дочент кафедры неорганической химии Дагестанского государственного университета. E-mail: ugga74@mail.ru.

ХАДИЖАТ МАГОМЕДОВНА ГАСАНОВА - к.х.н.,, доиент кафедры неорганической химии Дагестанского государственного университета. E-mail: hmgas@mail.ru

367000, Россия, Республика Дагестан, Махачкала, ул. Гаджиева, д. 43-а. Дагестанский государственный университет.

Приведены результаты по анализу математической модели кинетики возникновения химических осиилляций в гомогенной системе иистеин - оксигенированные комплексы кобальта (II) с о-дисалицилиденфенилендиамином и цитозином. На основе качественного анализа модели, представленного в виде системы из двух нелинейных дифференциальных уравнений, установлено, что реализуется одно стационарное состояние типа «неустойчивый фокус». Из этой особой точки возможна бифуркация в предельный иикл (бифуркация типа Андронова-Хопфа), что указывает на реализацию автоколебательного режима. Численное интегрирование модели показало, что она имеет решение в виде предельного иикла и процесс протекает в колебательном режиме при концентрациях иистеина и катализатора, соизмеримых с их экспериментальными величинами.
\end{abstract}

Ключевые слова: цистеин, комплексы кобальта (II), кинетика, осцилляция, модель, анализ.

\section{A MATHEMATICAL MODEL OF AUTOOSCILLATIONS IN THE SYSTEM OF CYSTEINE-OXYGENATED COBALT (II) COMPLEXES WITH $o$-DISALICYLIDENEPHENILENEDIAMINE AND CYTOSINE}

\footnotetext{
S.S. Etmisheva, U.G. Magomedbekov, U.G. Gasangadzhieva, Kh.M. Gasanova

Dagestan State University, 43-a, Gadzhieva st., Makhachkala, Republic of Dagestan, Russia

Results of the analysis of the mathematical model of kinetics of the occurrence of chemical oscillations in a homogeneous system cysteine - oxygenated cobalt (II) complexes with o-disalicylidenephenylenediamine and cytosine are presented. Based on the qualitative analysis of the model, presented as a system of two nonlinear differential equations, it has been established that one stationary state of the "unstable focus" type is realized. From this singular point, a bifurcation into a limit cycle (bifurcation of the Andronov-Hopf type) is possible, which indicates the realization of the self-oscillatory mode. The numerical integration of the model showed that it has a solution in the form of a limiting cycle and the process proceeds in an oscillatory mode at cysteine and catalyst concentrations comparable with their experimental values.
}

Key words: cysteine, cobalt (II) complexes, kinetics, oscillation, model, analysis. 


\section{Введение}

Интерпретация экспериментальных результатов по исследованию химических осцилляций и проведение анализа особенностей протекания колебательных химических реакций основаны на принципах нелинейной динамики, неравновесной термодинамики и математического моделирования $[1,2]$. Использование современных методов математического моделирования при рассмотрении критических явлений в химической кинетике позволяет решать широкий круг вопросов, связанных, прежде всего, с конкретизацией влияния управляющих параметров на механизм протекающих процессов [3].

Нами экспериментально установлено [4], что процесс гомогенного окисления цистеина (Cys, $R)$ в присутствии в качестве катализатора (cat) координационных соединений кобальта (II) с о-дисалицилиденфенилен-диамином (ДСФДА), цитозином $(C y t)$ и молекулярным кислородом протекает в колебательном режиме.

В настоящей статье представлены результаты по составлению и анализу математической модели кинетических закономерностей возникновения химических осцилляций в исследуемой системе.

\section{Обоснование и составление математической модели}

Построение и анализ математической модели кинетики процессов, протекающих в исследуемой системе, проводились на основе известного алгоритма [3, 5-7], включающего: составление кинетической схемы на основе элементарных стадий протекающих процессов; представление математической модели в виде системы автономных дифференциальных уравнений; упрощение и преобразование математической модели к безразмерному виду; проведение анализа и исследования полученной математической модели и интерпретация решения в терминах кинетической модели; сравнение полученных результатов с экспериментальными данными.

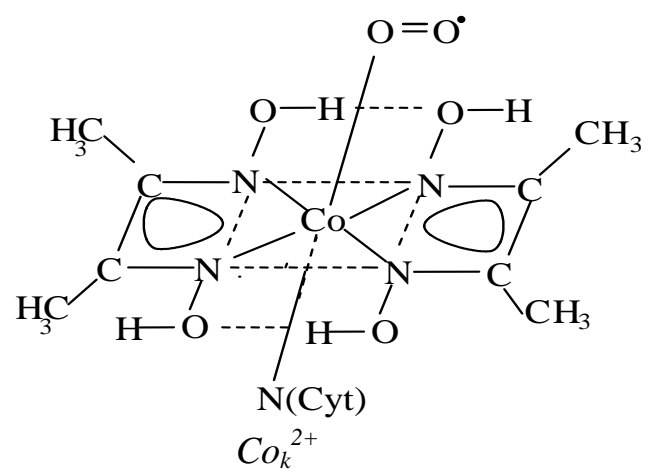

Формирование механизма гомогенного окисления цистеина в присутствии оксигенированных комплексов кобальта (II) с ДСФДА и Cyt проведено исходя из литературных данных [8-15] и некоторых предположений. Рассмотрение окислительно-восстановительных процессов, протекающих в исследуемой среде проводилось при $\mathrm{pH}>7$, следовательно, при этих условиях происходит ионизация по кислотной группе и реагент находится в виде $R_{S H}^{-}$за счет диссоциации по кислотной группе [8]. На образование при окислении цистеина промежуточных форм типа $R S^{-}$и $R S O H^{-}$указано в работах [8-10], а на возможность образования частиц типа $\mathrm{HO}_{2}{ }^{-}$и $\mathrm{HO}_{2}^{-}$отмечено в работе [9]. Координационные соединения переходных элементов в низших степенях окисления при соответствующем координационном окружении обратимо присоединяют молекулярный кислород, образуя форму $\mathrm{Me}_{\mathrm{k}} \mathrm{O}_{2}{ }^{2+}\left(\mathrm{Me}_{\mathrm{k}}-\right.$ связанные в комплекс ионы $\mathrm{Mn}^{2+}, \mathrm{Fe}^{2+}$ или $\mathrm{Co}^{2+}$, а связанный в комплекс кислород выступает в качестве окислителя) [5, 11-15].

С учетом этих фактов предложена схема протекающих окислительно-восстановительных процессов в исследуемой системе в следующем виде:

1. $\mathrm{RSH}^{-}+\mathrm{Co}_{k} \mathrm{O}_{2}^{2+} \stackrel{k_{1}}{\longrightarrow} \mathrm{RS}^{\bar{\bullet}}+\mathrm{HO}_{2}^{\bullet}+\mathrm{Co}_{k}{ }^{2+}$

2. $\mathrm{RSH}^{-}+\mathrm{HO}_{2}^{\bullet} \stackrel{k_{2}}{\longrightarrow} \mathrm{RS}^{-}+\mathrm{H}_{2} \mathrm{O}_{2}$

3. $\mathrm{RS}^{\overline{\boldsymbol{}}}+\mathrm{HO}_{2}^{\bullet \stackrel{k_{3}}{\longrightarrow}} \mathrm{RSOH}^{-}+1 / 2 \mathrm{O}_{2}$

4. $\mathrm{RSOH}^{-}+\mathrm{HO}_{2}^{\bullet} \stackrel{\mathrm{k}_{4}}{\longrightarrow} \mathrm{RS}^{-}+\mathrm{H}_{2} \mathrm{O}+\mathrm{O}_{2}$

5. $\mathrm{RS}^{\overline{\boldsymbol{}}}+\mathrm{H}_{2} \mathrm{O}+\mathrm{O}_{2} \stackrel{\mathrm{k}_{5}}{\longrightarrow} \mathrm{RSOH}^{-}+\mathrm{HO}_{2}^{\bullet}$

6. $\mathrm{RSOH}^{-}+\mathrm{RSOH}^{-} \stackrel{\mathrm{k}_{6}}{\longrightarrow} 2 \mathrm{RS}^{-}+\mathrm{H}_{2} \mathrm{O}+1 / 2 \mathrm{O}_{2}$

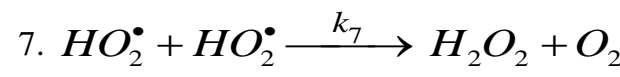

8. $\mathrm{Co}_{k}^{2+}+\mathrm{O}_{2} \stackrel{k_{8}}{\longrightarrow} \mathrm{Co}_{k} \mathrm{O}_{2}^{2+}$

9. $\mathrm{H}_{2} \mathrm{O}_{2} \stackrel{k_{9}}{\longrightarrow} \mathrm{H}_{2} \mathrm{O}+1 / 2 \mathrm{O}_{2}$,

где $\mathrm{RSH}^{-}, \mathrm{RS}^{-}$и $\mathrm{RSOH}^{-}$- различные формы превращений цистеина, $\mathrm{Co}_{k}{ }^{2+}$ и $\mathrm{Co}_{k} \mathrm{O}_{2}{ }^{2+}-$ неоксигенированные и оксигенированные формы комплексов кобальта (II), имеющие следующее строение:

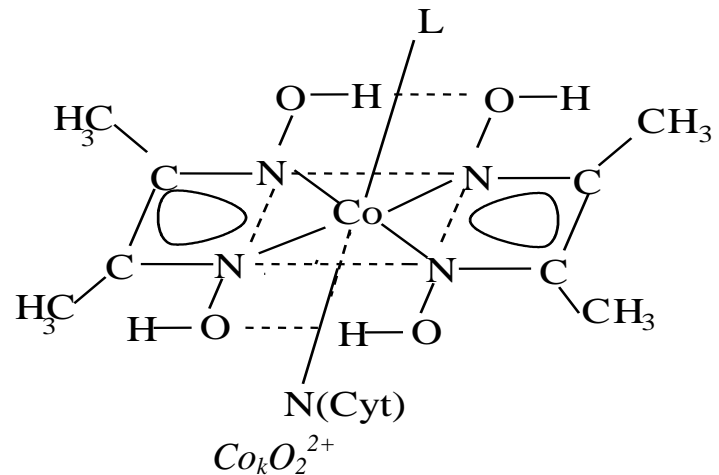

(L - лиганд, N(Cyt) - атом азота цитозина) 
В соответствии с этим алгоритмом кинетика сложной химической реакции, отвечающая определенной заданной схеме превращений в виде следующих уравнений $(1)[3,16,17]$.

$$
v_{\mathrm{ir}} X_{\mathrm{i}} \stackrel{\mathrm{k}_{\mathrm{r}}}{\longrightarrow} v_{\mathrm{jr}} \mathrm{X}_{\mathrm{j}}, \mathrm{i}, \mathrm{j}=1 \ldots \mathrm{N} ; \mathrm{r}=1 \ldots \mathrm{R}
$$

описывается системой дифференциальных уравнений в виде (2) [16, 17]:

$$
\frac{\mathrm{d}\left[\mathrm{X}_{\mathrm{i}}\right]}{\mathrm{dt}}=\sum_{\mathrm{r}=1}^{\mathrm{R}}\left(v_{\mathrm{ir}}^{\prime}-v_{\mathrm{ir}}\right) \mathrm{k}_{\mathrm{r}} \prod_{\mathrm{i}}\left[\mathrm{X}_{\mathrm{i}}\right]^{v_{\mathrm{ir}}}
$$

где $\mathrm{X}_{\mathrm{i}}$ - вещества, $v_{\text {ir }}$ - стехиометрические коэффициенты; $v_{\text {ir }}=0$, если $X_{\mathrm{i}}$ не входит в число веществ, вступающих в реакцию на r-й стадии и $v_{\mathrm{ir}}^{\prime}=0$, если веществ нет среди продуктов, образующих на r-й стадии.

Полученная на основе соотношения (2) система обыкновенных дифференциальных уравнений соответствует математической модели исследуемого процесса, поэтому изучение эволюции химических систем сводится к качественному анализу рассматриваемой модели и (или) её решению аналитическим путем или численными методами.

Дифференциальные уравнения, которые учитывают изменение концентрации всех компонентов реакционной среды во времени, выведенные в соответствии с кинетической схемой процесса (3), имеют следующий вид:

1. $d\left[\mathrm{RSH}^{-}\right] / d t^{\prime}=-k_{1}\left[\mathrm{RSH}^{-}\right]\left[\mathrm{Co}_{k} \mathrm{O}_{2}{ }^{2+}\right]-k_{2}\left[\mathrm{RSH}^{-}\right]\left[\mathrm{HO}_{2}{ }^{\circ}\right]$

2. $d\left[\mathrm{Co}_{k} \mathrm{O}_{2}{ }^{2+}\right] / d t^{\prime}=-k_{1}\left[\mathrm{RSH}^{-}\right]\left[\mathrm{Co}_{k} \mathrm{O}_{2}{ }^{2+}\right]+k_{8}\left[\mathrm{Co}_{k}{ }^{2+}\right]\left[\mathrm{O}_{2}\right]$

3. $d\left[R S^{-}\right] / d t^{\prime}=k_{1}\left[\mathrm{RSH}^{-}\right]\left[\mathrm{Co}_{k} \mathrm{O}_{2}{ }^{2+}\right]-k_{3}\left[\mathrm{RS}^{-}\right]\left[\mathrm{HO}_{2}{ }^{\circ}\right]+$

$$
\begin{gathered}
+k_{4}\left[\mathrm{RSOH}^{-}\right]\left[\mathrm{HO}_{2}^{-}\right]-k_{5}\left[\mathrm{RS}^{-}\right]\left[\mathrm{H}_{2} \mathrm{O}_{2}\right]\left[\mathrm{O}_{2}\right]+ \\
+2 k_{6}\left[\mathrm{RSOH}^{-}\right]\left[\mathrm{RSOH}^{-}\right]
\end{gathered}
$$

4. $d\left[\mathrm{HO}_{2}{ }^{\cdot}\right] / d t^{\prime}=k_{1}\left[\mathrm{RSH}^{-}\right]\left[\mathrm{Co}_{k} \mathrm{O}_{2}^{2+}\right]-k_{2}\left[\mathrm{RSH}^{-}\right]\left[\mathrm{HO}_{2}{ }^{-}\right]-$

$$
\begin{gathered}
-k_{3}\left[\mathrm{RS}^{-}\right]\left[\mathrm{HO}_{2}^{\circ}\right]-k_{4}\left[\mathrm{RSOH}^{-}\right]\left[\mathrm{HO}_{2}^{\cdot}\right]+ \\
+k_{5}\left[\mathrm{RS}^{-}\right]\left[\mathrm{H}_{2} \mathrm{O}_{2}\right]\left[\mathrm{O}_{2}\right]-k_{7}\left[\mathrm{HO}_{2}^{\cdot}\right]^{2}
\end{gathered}
$$

5. $d\left[\mathrm{Co}_{k}{ }^{2+}\right] / d t^{\prime}=k_{1}\left[\mathrm{RSH}^{-}\right]\left[\mathrm{Co}_{k} \mathrm{O}_{2}{ }^{2+}\right]-k_{8}\left[\mathrm{Co}_{k}{ }^{2+}\right]\left[\mathrm{O}_{2}\right]$

6. $d\left[\mathrm{H}_{2} \mathrm{O}_{2}\right] / d t^{\prime}=k_{2}\left[\mathrm{RSH}^{-}\right]\left[\mathrm{HO}_{2}^{\cdot}\right]+k_{4}\left[\mathrm{RSOH}^{-}\right]\left[\mathrm{HO}_{2}^{-}\right]+$

$$
\left.+k_{7}\left[\mathrm{HO}_{2}\right]^{2}\right]^{2}-k_{9}\left[\mathrm{H}_{2} \mathrm{O}_{2}\right]
$$

7. $d\left[\mathrm{RSOH}^{-}\right] / d t^{\prime}=k_{3}\left[\mathrm{RS}^{-}\right]\left[\mathrm{HO}_{2}^{\cdot}\right]-k_{4}\left[\mathrm{RSOH}^{-}\right]\left[\mathrm{HO}_{2}^{\cdot}\right]+$

$$
+k_{5}\left[\mathrm{RS}^{-}\right]\left[\mathrm{H}_{2} \mathrm{O}_{2}\right]\left[\mathrm{O}_{2}\right]-k_{6}\left[\mathrm{RSOH}^{-}\right]\left[\mathrm{RSOH}^{-}\right]
$$

8. $d\left[\mathrm{O}_{2}\right] / d t^{\prime}=k_{3}\left[\mathrm{RS}^{-}\right]\left[\mathrm{HO}_{2}{ }^{-}\right]-k_{4}\left[\mathrm{RSOH}^{-}\right]\left[\mathrm{HO}_{2}^{\circ}\right]+$

$$
+k_{5}\left[\mathrm{RS}^{-}\right]\left[\mathrm{H}_{2} \mathrm{O}_{2}\right]\left[\mathrm{O}_{2}\right]+k_{6}\left[\mathrm{RSOH}^{-}\right]\left[\mathrm{RSOH}^{-}\right]+
$$$$
+k_{7}\left[\mathrm{HO}_{2}{ }^{\circ}\right]-k_{8}\left[\mathrm{Co}_{k}{ }^{2+}\right]\left[\mathrm{O}_{2}\right]+k_{9}\left[\mathrm{H}_{2} \mathrm{O}_{2}\right]
$$

9. $d\left[\mathrm{H}_{2} \mathrm{O}_{2}\right] / d t^{\prime}=k_{4}\left[\mathrm{RSOH}^{-}\right]\left[\mathrm{HO}_{2}^{\circ}\right]-k_{5}\left[\mathrm{RS}^{-}\right]\left[\mathrm{H}_{2} \mathrm{O}_{2}\right]\left[\mathrm{O}_{2}\right]+$

$$
+k_{6}\left[\mathrm{RSOH}^{-}\right]\left[\mathrm{RSOH}^{-}\right]+k_{9}\left[\mathrm{H}_{2} \mathrm{O}_{2}\right]
$$

Проведение анализа такого типа систем указанными методами, и, особенно, трактовка получаемых результатов, довольно затруднительно (система содержит девять нелинейных дифференциальных уравнений), поэтому было сделано ряд упрощений математической модели с целью умень- шения числа дифференциальных уравнений в системе (4).

При реконструкции динамики исследуемой системы по временным последовательностям данных получено [4], что размерность фазового пространства для всех условий эксперимента соответствует пяти. Вследствие этого получаем, что количество компонентов реакционной смеси, которое необходимо учитывать при анализе кинетического механизма и, следовательно, число дифференциальных уравнений в анализируемой модели также соответствует пяти.

Дальнейшая редукция системы дифференциальных уравнений была проведена с учетом следующих моментов. Во-первых, нам известны исходные концентрации реагента и катализатора, что позволяет, исходя из уравнений материального баланса, выразить через них концентрации веществ, в которые в ходе реакций они превращаются. Во-вторых, используя принцип квазистационарности Боденштейна - Темкина, можно предположить, что концентрации $\mathrm{H}_{2} \mathrm{O}_{2}, \mathrm{RSOH}^{-}$, а также различных форм катализатора будут приблизительно постоянными (это вытекает из стадий 2,7 и 9 - для $\mathrm{H}_{2} \mathrm{O}_{2}$, реакций $3,4,5$ и 6 - в случае частиц $\mathrm{RSOH}^{-}$и реакций 1 и 9 - для ионов $\mathrm{Co}_{k} \mathrm{O}_{2}{ }^{2+}$ и $\mathrm{Co}_{k}{ }^{2+}$ ). Можно считать постоянной концентрацию молекулярного кислорода (эксперимент проводится в открытой по отношению к воздуху ячейке [18] и концентрация кислорода определяется её растворимостью в реакционной среде).

После проведения этих упрощений число дифференциальных уравнений уменьшается до двух, и соответствующую математическую модель можно представить в следующем виде:

$$
\begin{gathered}
\frac{d C_{x}}{d t^{\prime}}=k_{1}\left(C_{a}-C_{x}\right) C_{b}-k_{3} C_{x} C_{y}+ \\
+k_{2}\left(C_{a}-C_{x}\right) C_{y}+k_{4} C_{y}-k_{5} C_{x}+2 k_{6} \\
\frac{d C_{y}}{d t^{\prime}}=k_{1}\left(C_{a}-C_{x}\right) C_{b}-k_{3} C_{x} C_{y}-k_{2}\left(C_{a}-\right. \\
\left.-C_{x}\right) C_{y}-k_{4} C_{y}+k_{5} C_{x}-k_{7} C_{y} C_{y}
\end{gathered}
$$

где $t^{\prime}$ - время, $C_{x}=\left[R S^{-}\right], C_{y}=\left[\mathrm{HO}_{2}{ }^{\circ}\right], C_{a}=C_{R(u c x)}$, $C_{b}=C_{\text {cat }}(u c x)$

Для проведения качественного анализа и численного решения систему (5) необходимо привести её к безразмерному виду. С этой целью введем новые обозначения:

$$
\begin{gathered}
x=\omega C_{X}, y=\eta C_{Y}, z=\gamma C_{Z}, t=\delta t^{\prime}, a=C_{A}, b=C_{b}, \\
\omega=k_{3} / k_{2}, \quad \eta=k_{2} / k_{1}, \delta=k_{2},
\end{gathered}
$$

тогда система кинетических уравнений (4) примет вид: 


$$
\begin{aligned}
& \frac{d x}{d t^{\prime}}=\frac{\omega}{\delta}\left[k_{1}\left(a-\frac{x}{\omega}\right) b+k_{2}\left(a-\frac{x}{\omega}\right) \frac{y}{\eta}-k_{3} \frac{x}{\omega} \frac{y}{\eta}+k_{4} \frac{y}{\eta}-k_{5} \frac{x}{\omega}+2 k_{6}\right] \\
& \frac{d y}{d t^{\prime}}=\frac{\eta}{\delta}\left[k_{1}\left(a-\frac{x}{\omega}\right) b-k_{2}\left(a-\frac{x}{\omega}\right) \frac{y}{\eta}-k_{3} \frac{x}{\omega} \frac{y}{\eta}-k_{4} \frac{y}{\eta}+k_{5} \frac{x}{\omega}-k_{7}\left(\frac{y}{\eta}\right)^{2}\right]
\end{aligned}
$$

Для приведения системы уравнений к наиболее простому безразмерному виду введем обозначения: $\varepsilon=k_{3} k_{1} / k_{2}^{2}, \mu=k_{2} / k_{3}, \rho=k_{4} / k_{2}, \varphi=k_{7} k_{1} / k_{2}^{2}$, $\gamma=k_{6} / k_{1}, \sigma=k_{5} k_{2} / k_{3} k_{1}$.

Тогда, проведя соответствующие преобразования, исследуемую систему дифференциальных уравнений (6) можно записать в следующем безразмерном виде:

$$
\begin{aligned}
& \frac{d x}{d t^{\prime}}=[(a-\mu x) b+(a-\mu x) y-x y+\rho y-\sigma x+2 \gamma] \varepsilon ; \\
& \frac{d y}{d t^{\prime}}=(a-\mu x) b-(a-\mu x) y-x y-\rho y+\sigma x-\varphi y^{2}(7)
\end{aligned}
$$

$\mathrm{C}$ целью установления особенностей динамики рассматриваемого сложного химического процесса необходимо провести в первую очередь качественный анализ системы из двух дифференциальных уравнений нелинейного типа, представленной в качестве математической модели.

\section{Качественный анализ математической модели}

Согласно существующей процедуре $[6,16$, 17] при проведении качественного анализа левые части дифференциальных уравнений в системе (7) приравниваем нулю и получаем систему алгебраических уравнений в следующем виде:

$$
\begin{array}{r}
{[(a-\mu x) b+(a-\mu x) y-x y+\rho y-\sigma x+2 \gamma]=0} \\
(a-x \mu) b-(a-\mu x) y-x y-\rho y+\sigma x-\varphi y^{2}=0(8) \\
\text { Можно показать, что исследуемая система }
\end{array}
$$

дифференциальных уравнений при $\gamma=\frac{a b \sigma}{\mu b-\sigma}$ имеет единственную неподвижную точку (стационарное состояние) с координатами $\bar{x}=\frac{a b+2 \gamma}{\mu b+\sigma}$; $\bar{y}=0$.

При анализе устойчивости стационарного состояния $[7,17]$ необходимо построить матрицу Рауса - Гурвица (9). Для обсуждаемого примера эта матрица имеет вид:

$$
\mathrm{A}=\left|\begin{array}{cc}
(-\mu b-\sigma)-\lambda & a-\mu x-x+\rho \\
-\mu+\sigma & -(a-\mu x)-x-\rho-\lambda
\end{array}\right|=0
$$

Характеристическое уравнение, при этом, представляется в виде:

$$
\lambda^{2}-T \lambda^{2}+\Delta \lambda=0
$$

где

$$
\begin{gathered}
T=\left[\left(\mu b+\sigma+a+\rho+\frac{a b+2 \gamma}{\mu b+\sigma}\right)-\mu \frac{a b+2 \gamma}{\mu b+\sigma}\right], \\
\text { и } \Delta=2 a \mu b+2 \mu b \rho+\frac{\left(2 \sigma-2 \mu^{2} b\right)(a b+2 \gamma)}{\mu b+\sigma} .
\end{gathered}
$$

Решения уравнения (10) при $T^{2}-4 \Delta<0$ в обозначениях $T$ и $\Delta$ представляются в виде:

$$
\lambda_{1}=\frac{T}{2}+\frac{\sqrt{T^{2}-4 \Delta}}{2} i \text { и } \lambda_{2}=\frac{T}{2}-\frac{\sqrt{T^{2}-4 \Delta}}{2} i
$$

Для случая, когда одно из решений уравнения (в нашем случае $\lambda_{2}$ ) имеет вид $\lambda_{2}=\frac{T}{2}-\frac{\sqrt{T^{2}-4 \Delta}}{2} i$, возможна реализация неустойчивого состояния с особой точкой типа «неустойчивый фокус» [16, 17]. Из этой особой точки возможна бифуркация в предельный цикл (бифуркация типа АндроноваХопфа), что является свидетельством возможности реализации в исследуемой системе химических неустойчивостей в виде возникновения химических осцилляций $[7,17]$.

\section{Численное интегрирование математической модели}

Следующим этапом анализа математической модели является численное решение системы дифференциальных уравнений. Это тем более важно, что результаты, получаемые на основе численного интегрирования, позволяют проверять заключение, сделанное об устойчивости системы и возникновении критических состояний методами качественного анализа.

Для численного интегрирования анализируемой системы обыкновенных дифференциальных уравнений, представленной в качестве математической модели протекающих процессов в колебательном режиме, использована программа Mathcad с подпрограммой Rkadapt [19].

Для выявления особенностей процесса гомогенного окисления цистеина расчетным путем определены пределы концентрации аминокислоты и катализатора, при которых возникают автоколебания. Для этого, согласно существующей процедуре, в качестве управляющих параметров определены исходные концентрации реагента - цистеина (a), а также катализатора - оксигенированных комплексов кобальта (II) с ДСФДА и $C y t(b)$; в качестве переменных были выбраны промежуточные вещества превращения цистеина и продукты неполного восстановления координированного и растворенного кислорода в виде: $x=\left[R S^{\boldsymbol{}}\right] ; y=\left[\mathrm{HO}_{2}^{\bullet}\right]$. (О возможности образования такого типа интермедиатов указано в монографии [10] и отмечено нами в работах $[20,21])$. 
Решения для различных значений концентраций реагента $(a)$ и оксигенированных комплексов кобальта (б) при $\left(\varepsilon=3,6 \cdot 10^{-4} ; \mu=16,5 ; \rho=8,0\right.$; $\sigma=3,0 \cdot 10^{-5} ; \gamma=8,0 \cdot 10^{-5} ; \varphi=3,0 \cdot 10^{5}$ представлены на рис.1 (a, б, в, г).

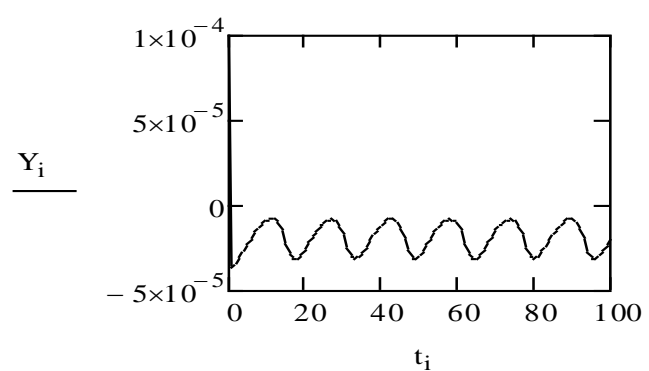

(I)

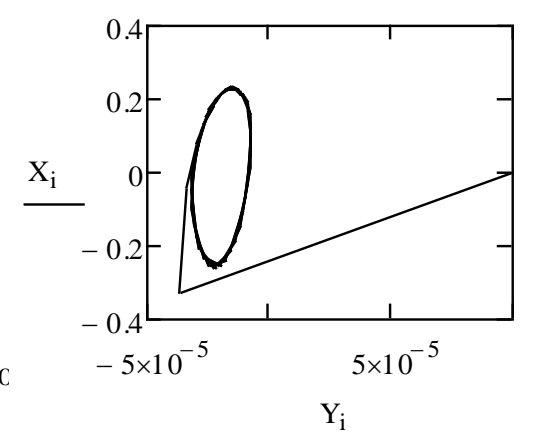

(II)

6
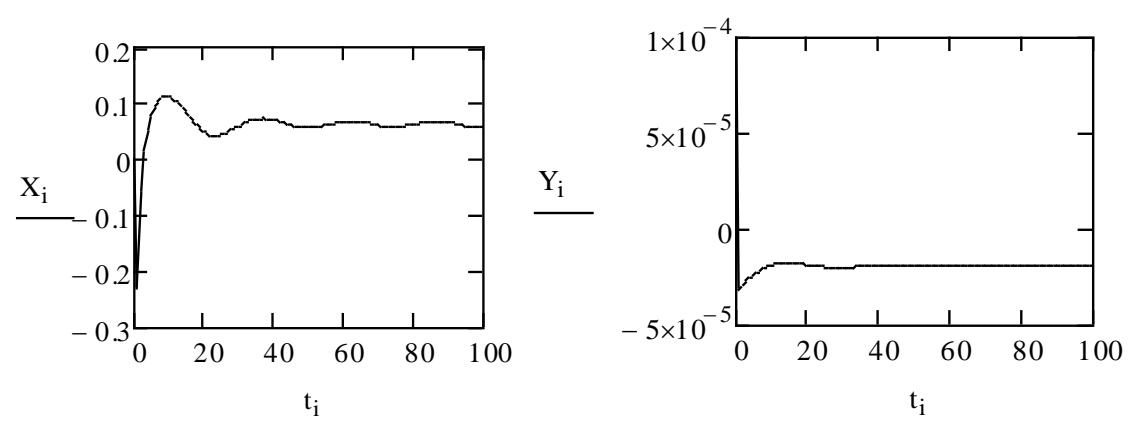

(I)

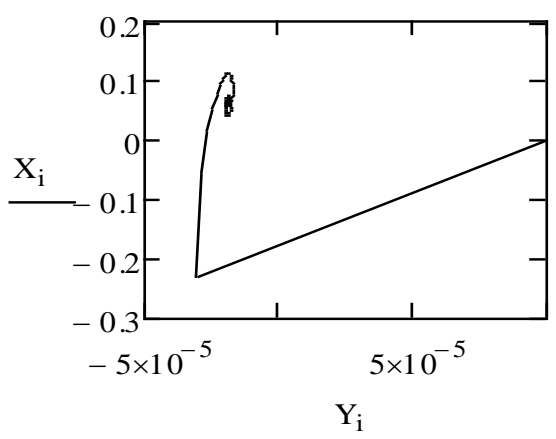

(II)

B

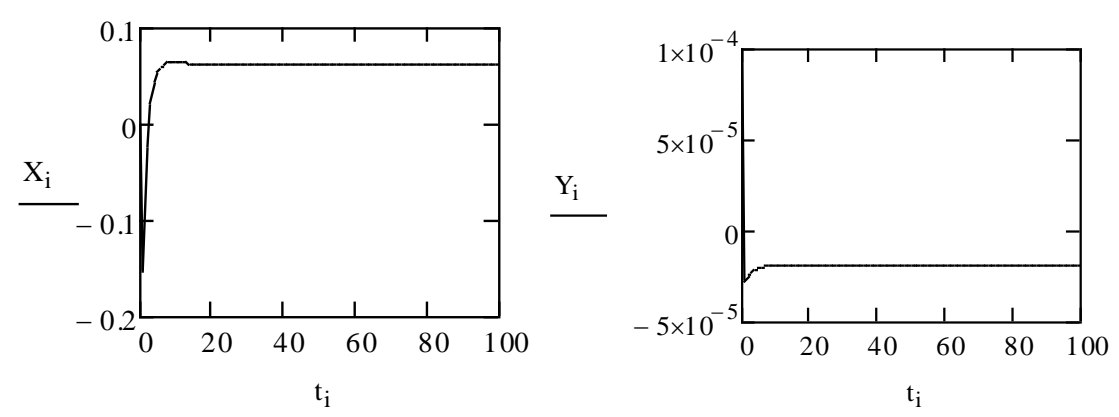

(I)

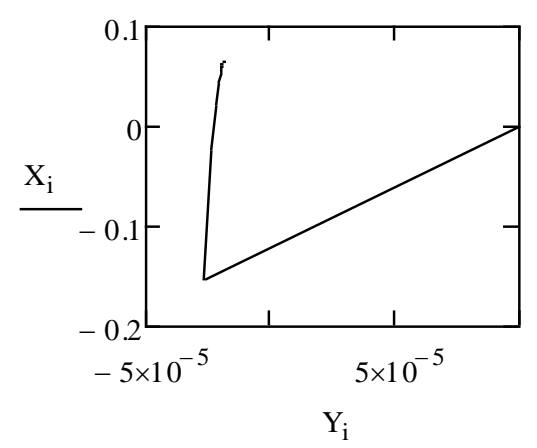

(II)

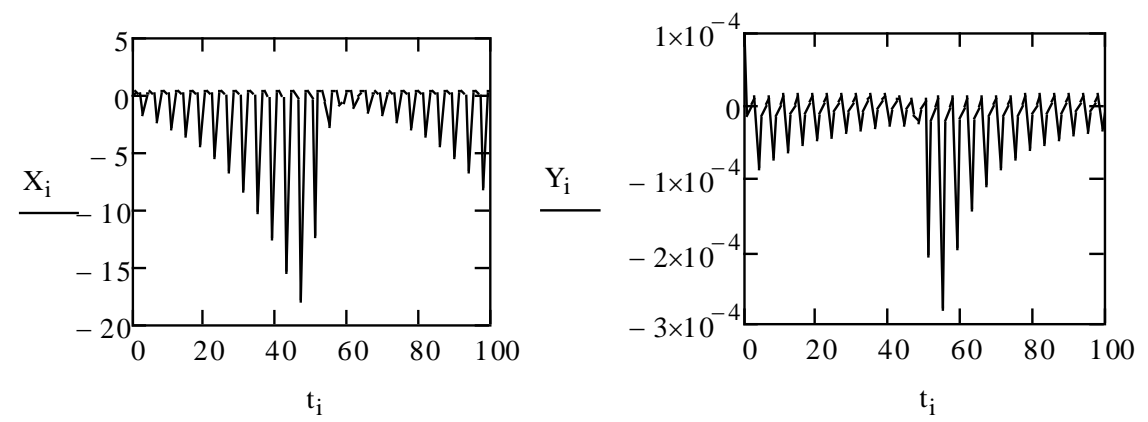

(I)

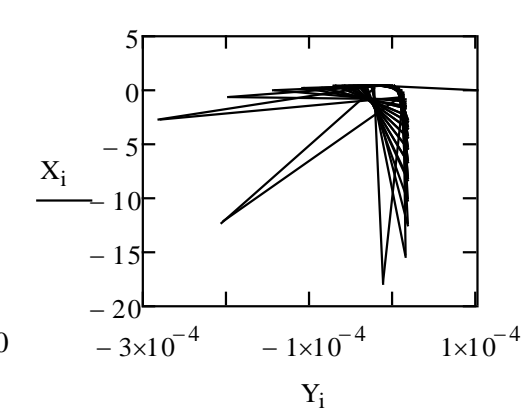

(II)

Рис. Зависимость изменения концентрации частиц $\mathrm{X}_{\mathrm{i}}=\left[\mathrm{RS}^{-}\right] ; \mathrm{Y}_{\mathrm{i}}=\left[\mathrm{HO}_{2}\right]$ от времени $\mathrm{t}_{\mathrm{i}}(\mathrm{I})$. Фазовый портрет систем в координатах $X_{\mathbf{i}}-\mathbf{Y}_{\mathrm{i}}$, (II)

a) $a=5,0 \cdot 10^{-4} ; b=2,5 \cdot 10^{-5} ;$ б) $a=5,0 \cdot 10^{-4} ; b=2,6 \cdot 10^{-5}$; в) $a=5,0 \cdot 10^{-4} ; b=2,7 \cdot 10^{-5} ;$ г) $a=5,0 \cdot 10^{-4} ; b=1,9 \cdot 10^{-5}$ 
Результаты расчетов, представленные на рисунке, показывают, что колебательные решения реализуются при концентрациях реагента и катализатора $\mathrm{C}_{\mathrm{cat}}=5,0 \cdot 10^{-4}$ и $\mathrm{C}_{\mathrm{b}}=(2,5 \div 2,6) \cdot 10^{-5}$ соответственно; эти величины сопоставимы с опытными значениями концентраций цистеина и оксигенированных комплексов кобальта (II), при которых возникают химические осцилляции.

Таким образом, можно заключить, что численное интегрирование обсуждаемой математической модели для различных начальных условий свидетельствует о том, что рассматриваемая математическая модель имеет решение в виде предельного цикла и процесс протекает в колебательном режиме при определенных исходных концентрациях цистеина и катализатора, сопоставимых с количествами, при которых экспериментально наблюдаются концентрационные колебания. Сравнение экспериментальных и расчетных данных позволяет констатировать, что обсуждаемая математическая модель удовлетворительно описывает процессы возникновения химических осцилляций при гомогенном окисления цистеина в присутствии оксигенированных комплексов кобальта (II) с о-дисалицилиденфенилендиамином и цитозином.

Работа выполнена на базе Научно-образовательного иентра ДГУ «Нелинейная химия» $c$ использованием оборудования Центра коллективного пользования ДГУ «Аналитическая спектроскопия».

\section{Лumepamypa}

1. Малинеикий Г.Г., Потапов А.Б., Подлазов А.В. Нелинейная динамика: Подходы, результаты, надежды. М.: Книжный дом «ЛИБРИКОМ». 2016. 280 с.

2. Кольиова Э.М., Третьяков Ю.Д., Гордеев Л.С., Вертегел A.A. Нелинейная динамика и термодинамика необратимых процессов в химии и химической технологии. М.: Химия. 2001. 408 с.

3. Быков В.И., Цыбенова С.Б. Нелинейные модели химической кинетики. М.: КРАСАНД. 2011. 400 с.

4. Етмишева С.С., Магомедбеков У.Г., Гасангаджиева У.Г. Изв. вузов. Химия и хим. технология. 2018. Т. 61. Вып. 8. С. 40-46; DOI: 10.6060/ivkkt.20186108.5765.
5. Магомедбеков У.Г. Окисление биосубстратов в колебательном режиме. Махачкала: ИПЦ ДГУ. 2002. $132 \mathrm{c}$.

6. Быков В.И. Моделирование критических явлений в химической кинетике. М.: КомКнига. 2006. 328 с

7. Мазуров М.E. Идентификация математических моделей нелинейных динамических систем. М.: ЛЕНАНД. 2019. $284 \mathrm{c}$.

8. Биохимия / Под ред. Е.С. Северина. М.: ГЭОТАР. $2004.779 \mathrm{c}$

9. Сычев А.Я., Исак В.Г. Успехи химии. 1995. Т. 64. №12. C.1183-1209.

10. Торчинский Ю.М. Сульфгидрильные и дисульфидные группы белков. М.: Наука. 1971. 228 с.

11. Братушко Ю.И. Координационные соединения $3 \mathrm{~d}$ переходных металлов с молекулярным кислородом. Киев: Наукова Думка. 1987. 168 с.

12. Магомедбеков У.Г., Етмишева С.С., Хасанов И.И. Вестник Чеченского государственного университета. 2013. Вып. 1. С. 116-120.

13. Магомедбеков У.Г. Гасангаджиева У.Г., Гасанова Х.М., Гаджибалаева 3.М., Магомедова Р.А. Известия Вузов. Сев.-Кавк. регион. Естественные науки. 2008. № 6 (146) С. 48-52.

14 Магомедбеков У.Г., Гасангаджиева У.Г., Гасанова Х.М., Магомедбеков Н.X. Вестник Дагестанского государственного университета. Ест. Науки. 2010. Вып. 6. С. 112-114.

15. Магомедбеков У.Г., Гасангаджиева У.Г., Гасанова Х.М., Магомедбеков Н.Х. Вестник Моск. ун-та. Сер.2. Химия. 2011. Т.52. №6. С.443-448.

16. Самарский А.А., Михайлов А.П. Математическое моделирование. М.: Наука. 1997. 320 с.

17. Немыцикий В.В., Степанов В.В. Качественная теория дифференциальных уравнений М.: ЛЕНАНД. 2017. $552 \mathrm{c}$.

18. Досон Р., Эллиот Д., Эллиот У., Джонс К. Справочник биохимика. М. Мир. 1991. 554 с.

19. Pojman J.A. Studying Nonlinear Chemical Dynamics with Numerical Experiments. Department of Chemistry \& Biochemistry. University of Southern Mississippi. 1997. P. 339-348.

20. Магомедбеков У.Г., Гасангаджиева У.Г., Гасанова Х.М. Вестник Моск. Ун-та. Сер. 2. Химия. 2013. T. 54. № 6. C. 330-341.

21. Магомедбеков У.Г., Гасангаджиева У.Г., Гасанова Х.М., Магомедбеков Н.Х. Рос. хим. ж. (Ж. Рос. хим. об-ва им. Д.И. Менделеева). 2009. Т. 56. № 6. С. 74. 